\title{
Parathyroid hormone response to severe vitamin D deficiency is associated with femoral neck bone mineral density: an observational study of $\mathbf{4 0 5}$ women with hip-fracture
}

\author{
Marco Di Monaco, Carlotta Castiglioni, Rosa Tappero
}

Osteoporosis Research Center and Division of Physical Medicine and Rehabilitation, Presidio Sanitario San Camillo, Fondazione Opera San Camillo, Torino, Italy

\begin{abstract}
OBJECTIVE: Hip-fracture patients with vitamin D deficiency can have either secondary hyperparathyroidism or normal levels of parathyroid hormone (PTH). We hypothesized that bone mineral density (BMD) could be lower in patients with high PTH levels than in those with normal levels of PTH, irrespectively of the severity of vitamin D depletion. DESIGN: In this cross-sectional study, we examined 405 women who had serum 25 -hydroxyvitamin $D$ below $12 \mathrm{ng} / \mathrm{ml} \mathrm{20.0} \pm 5.9$ (mean $\pm \mathrm{SD})$ days after a hip-fracture. PTH was assessed by a chemiluminescent immunometric assay and BMD by dual-energy $x$-ray absorptiometry at the unfractured femoral neck. RESULTS: BMD was significantly lower in the 148 women with secondary hyperparathyroidism than in the 257 with normal PTH levels: the mean T-score (SD) was $\mathbf{- 2 . 8 8}$ (0.93) and $-2.65(0.83)$, respectively, in the two groups (mean difference $0.23 ; 95 \%$ CI $0.05-0.41$; $P=0.010)$. The association between PTH status and BMD persisted after adjustment for age, body mass index, phosphate, albumin-adjusted total calcium, 25-hydroxyvitamin $D$, estimated glomerular filtration rate, and magnesium $(\mathrm{P}=0.01)$. The presence of secondary hyperparathyroidism was significantly associated with a femoral neck T-score lower than $\mathbf{- 2 . 5}$. The adjusted odds ratio was $1.81(95 \%$ CI $1.11-2.95 ; P=0.017)$. CONCLUSIONS: Our results show that PTH levels in the presence of severe vitamin $D$ deficiency were significantly associated with femoral BMD in women with hip-fracture. Prevention and treatment of vitamin D deficiency may be particularly relevant in women who develop secondary hyperparathyroidism.
\end{abstract}

Key words: Hip-fracture, Parathyroid hormone, Secondary hyperparathyroidism, Vitamin D

Address for correspondence:

Dr. Marco Di Monaco; Osteoporosis Research Center and Division of Physical Medicine and Rehabilitation, Presidio Sanitario San

Camillo, Fondazione Opera San Camillo, Strada Santa Margherita

136, 10131, Torino, Italy; Tel.: +39 011 8199411;

Fax:+39011 8193012, E-mail: (i) marco.di.monaco@alice.it;

(ii) m.di-monaco@h-sancamillo.to.it

Received: 04-08-2016, Accepted: 28-11-2016

\section{INTRODUCTION}

The beneficial effects of vitamin D on bone tissue have long been known and have been attributed to the role of its active metabolites in the intestinal absorption of calcium and in the process of bone 
mineralization. ${ }^{1}$ Recently, numerous extra-skeletal effects of vitamin D have been shown, including actions on neuromuscular and cognitive functions which can reduce the risk of falling. ${ }^{2,3}$ Overall, vitamin D is thought to exert favorable effects on both bone fragility and fall risk, i.e., the two major risk factors for osteoporotic fractures in older people. As a consequence, the administration of vitamin $\mathrm{D}$ is recommended worldwide to reduce the risk of fractures. ${ }^{4}$ However, substantial proportions of older people are still affected by vitamin D deficiency ${ }^{5,6}$ and very low serum levels of 25 -hydroxyvitamin D are common at the time of hospitalization for a fracture of the hip, ${ }^{7-10}$ with no apparent improvements in the last decade. ${ }^{11}$

Parathyroid hormone (PTH) excess can contribute to bone $\operatorname{loss}^{12}$ and possibly to fall risk ${ }^{13}$ in patients with vitamin $\mathrm{D}$ depletion because the latter is an established cause of secondary hyperparathyroidism. ${ }^{14,15}$ However, PTH elevation is not always found in vitamin D depletion: several patients with severe vitamin D deficiency do not have PTH excess, as first shown by Sahota et al. ${ }^{16}$ and confirmed by several recent reports. ${ }^{17-20}$ At present, the reasons why vitamin $\mathrm{D}$ depletion leads to either secondary hyperparathyroidism or normal PTH levels in individual subjects are not clear. ${ }^{16-20} \mathrm{In}$ any case, the PTH response to vitamin D depletion may result in different levels of bone loss. ${ }^{16}$

Our aim was to study the relationship between PTH values and bone mineral density (BMD) in hip-fracture women with vitamin D depletion. We hypothesized that women with secondary hyperparathyroidism could have lower BMD levels than those with normal PTH levels.

\section{METHODS}

\section{Patients and setting}

We retrospectively evaluated 730 Caucasian women with a hip-fracture, admitted consecutively to our Physical Medicine and Rehabilitation division. Our hospital is in Torino, Italy, a city with about one million inhabitants and the 730 women came from several orthopedic wards in various hospitals. All the women were referred for acute inpatient rehabilitation by the consultant physiatrists of the orthopedic wards.
The criteria agreed on for selecting women with hipfractures to undergo acute inpatient rehabilitation were as follows: (1) health conditions allowing a total of three hours of physical therapy and/or occupational therapy daily; (2) weight-bearing to tolerance on the fractured hip; and (3) a potential high increase in ability to function in activities of daily living as a result of an intensive rehabilitation regimen. A total of 31 of the 730 women we evaluated were excluded from our study because their hip-fracture was caused by either major trauma or cancer affecting bone. The remaining 699 women had sustained fractures that either were spontaneous or resulted from minimal trauma (trauma equal to or less than a fall from a standing position). Nine of these 699 women were excluded from our study because of either albuminadjusted serum levels of calcium exceeding $11 \mathrm{mg} /$ $\mathrm{dl}$ or low estimated glomerular filtration rate (GFR $<15 \mathrm{ml} / \mathrm{min}$ ). Twelve women were excluded because they could not undergo dual-energy x-ray absorptiometry (DXA) assessment due to either refusal or presence of an arthroplasty at the non-fractured hip. Eight patients were excluded because of missing data. Among the remaining 670 women we focused on the 405 with serum levels of 25 -hydroxyvitamin $\mathrm{D}$ below 12ng/ml. Institutional Review Board approval was obtained for the study protocol.

\section{Outcome Measures}

A blood sample was collected during the first three days of hospitalization, $20.0 \pm 5.9$ days (mean $\pm \mathrm{SD}$ ) after fracture occurrence, in the morning after an overnight fast. In each subject we evaluated 25 -hydroxyvitamin $\mathrm{D}$ by an immunoenzymatic assay (coefficient of variation intrassay $<8 \%$; interassay $<10 \%$ ) (IDS Inc., Fountain Hills, AZ, USA), PTH by two-site chemiluminescent enzyme-labelled immunometric assay (coefficient of variation intraassay 5.7\%, interassay 8.8\%) (DPC Inc., Los Angeles, CA, USA), total calcium (by a photometric color test), phosphate, albumin, magnesium, and creatinine. GFR was estimated by the 4-variable Modification of Diet in Renal Disease (MDRD) Study equation.

In all 405 women we assessed BMD at the nonfractured femoral neck by DXA (Hologic Discovery bone densitometer, USA) on the same day as the blood sample collection. The results were recorded as 
T-scores (the reference population was derived from the third National Health and Nutrition Examination Survey). The coefficient of variation within subjects calculated from two repeated measurements with repositioning was $1 \%$. Body weight and height were measured in each subject and body mass index (BMI) was calculated as weight/height ${ }^{2}$.

None of the patients included in the study had begun specific treatment with drugs for osteoporosis after fracture occurrence before the DXA and laboratory assessment.

\section{Data Analyses}

The 405 women were divided into two groups according to their PTH levels: secondary hyperparathyroidism was diagnosed with high PTH levels $(>75 \mathrm{pg} / \mathrm{ml})$, whereas levels $\leq 75 \mathrm{pg} / \mathrm{ml}$ where defined as normal. Comparisons between the two groups of women were performed by a Student $T$ test for the continuous variables, which were normally distributed based on a Shapiro-Wilk test (i.e., age, BMI, and BMD) and on a Mann-Whitney $U$ test for the continuous variables which were non-normally distributed (i.e., PTH, phosphate, albumin-adjusted total calcium, 25-hydroxyvitamin D, estimated GFR, and magnesium). Additionally, femoral BMD expressed as a T-score was included in a standard linear multiple regression model as the dependent variable. The regression model included the following eight independent variables: presence of either secondary hyperparathyroidism or normal PTH levels, age, BMI, phosphate, albumin-adjusted total calcium, 25-hydroxyvitamin D, estimated GFR, and magnesium. The residuals were normally distributed in the regression model. Homoscedasticity was verified by plotting the residuals against the predicted values: the variance of the residuals looked homogeneous across levels of the predicted values. Collinearity diagnostics showed that the percent of variance in each predictor that could not be accounted for by the other predictors was always greater than $90 \%$ (no redundant predictors were found).

A binary logistic regression test was used to adjust the association between PTH category (either elevated or normal) and a femoral neck T-score lower than -2.5 for the seven potential confounders listed above.

The statistical package used was SPSS, version 14. The significance threshold was set at 0.05 .

\section{RESULTS}

One hundred forty-eight of the 405 women (37\%) had secondary hyperparathyroidism (PTH serum levels exceeding $75 \mathrm{pg} / \mathrm{ml}$ ), whereas the remaining 257 (63\%) had normal PTH levels despite severe vitamin $\mathrm{D}$ deficiency. Table 1 shows the descriptive characteristics of the two groups of women and the between-group comparisons. Mean BMD levels were significantly lower in the women with secondary hyperparathyroidism than in those with normal PTH

Table 1. Comparisons between the women with functional hypoparathyroidism and those with secondary hyperparathyroidism

\begin{tabular}{lccc}
\hline Variable & $\begin{array}{c}\text { Functional hypoparathyroidism } \\
(\mathbf{N}=\mathbf{2 5 7})\end{array}$ & $\begin{array}{c}\text { Secondary hyperparathyroidism } \\
\mathbf{( N = 1 4 8 )}\end{array}$ & P \\
\hline Age (years), mean (SD) & $78.7(8.1)$ & $82.4(6.7)$ & $<0.001$ \\
Body Mass Index (kg/m²), mean (SD) & $22.9(4.1)$ & $23.6(4.2)$ & 0.131 \\
PTH (pg/ml) & $47(34-61)$ & $107(85-139)$ & $<0.001$ \\
Phosphate (mg/dl) & $3.7(3.3-4.1)$ & $3.4(3.1-3.8)$ & $<0.001$ \\
Albumin-adjusted calcium (mg/dl) & $9.0(8.2-9.5)$ & $8.9(7.8-9.5)$ & 0.114 \\
25-hydroxyvitamin D (ng/ml) & $6.8(3.8-9.0)$ & $7.0(4.0-8.9)$ & 0.778 \\
Estimated GFR (ml/min) & $74.5(63.8-87.8)$ & $71.1(56.0-84.3)$ & $<0.001$ \\
Magnesium (mg/dl) & $2.0(1.9-2.1)$ & $2.0(2.0-2.1)$ & 0.415 \\
BMD (T-score), mean (SD) & $-2.65(0.83)$ & $-2.88(0.93)$ & 0.010 \\
BMD (g/cm²), mean (SD) & $0.55(0.09)$ & $0.52(0.10)$ & 0.010 \\
\hline Da
\end{tabular}

Data are shown as median and interquartile range where not otherwise stated. 
levels: the mean T-score (SD) was $-2.88(0.93)$ and -2.65 (0.83), respectively, in the two groups (mean difference $0.23 ; 95 \%$ CI $0.05-0.41 ; \mathrm{P}=0.010$ ). The results of linear multiple regression for BMD measured at the femoral neck are shown in Table 2: three of the eight independent variables included in the standard regression model (i.e. PTH status, BMI, and age) were significantly associated with the dependent variable (femoral BMD expressed as a T-score). In particular, the presence of secondary hyperparathyroidism was negatively associated with BMD $(\mathrm{p}=0.001)$. A low BMD value (femoral neck T-score lower than -2.5) was found in 157 of the 257 women with normal PTH levels (i.e., 61\%), whereas it was observed in 103 of the 148 women with secondary hyperparathyroidism (i.e., 70\%). After multiple adjustments, the presence of secondary hyperparathyroidism was significantly associated with a femoral neck T-score lower than -2.5 . The adjusted odds ratio was 1.81 (95\% CI 1.11 - 2.95; $\mathrm{P}=0.017$ ), as shown in Table 3.

\section{DISCUSSION}

Data show that PTH response to severe vitamin $\mathrm{D}$ deficiency was significantly associated with BMD assessed at the femoral neck after a hip-fracture:

Table 2. Linear multiple regression analysis model

\begin{tabular}{lccc}
\hline Independent variables & B (95\% CI) & Beta & P \\
\hline (Constant) & $-2.81(-4.52 ;-1.10)$ & 0.001 \\
PTH status (either secondary hyperparathyroidism or functional hypoparathyroidism) & $-0.28(-0.46 ;-0.11)$ & -0.16 & 0.001 \\
Age (years) & $-0.01(-0.02 ; 0)$ & -0.10 & 0.043 \\
Body Mass Index (kg/m²) & $0.08(0.06 ; 1.0)$ & 0.36 & $<0.001$ \\
Phosphate (mg/dl) & $-0.02(-0.16 ; 0.11)$ & -0.01 & 0.774 \\
Albumin-adjusted calcium (mg/dl) & $-0.05(-0.13 ; 0.03)$ & -0.06 & 0.216 \\
25-hydroxyvitamin D (ng/ml) & $0.01(-0.02 ; 0.04)$ & 0.03 & 0.442 \\
Estimated GFR (ml/min) & $-0.003(-0.01 ; 0.00)$ & -0.08 & 0.079 \\
Magnesium (mg/dl) & $0.001(-0.01 ; 0.01)$ & 0.01 & 0.895 \\
\hline
\end{tabular}

The dependent variable was femoral bone mineral density expressed as a T-score. The independent variables were those listed in the Table. For each independent variable, unstandardized B coefficients with $95 \%$ confidence intervals, standardized Beta coefficients, and $\mathrm{P}$ values are shown. Secondary hyperparathyroidism was conventionally attributed a value of 1 (functional hypoparathyroidism was conventionally attributed a value of 0$)$. $\mathrm{R}^{2}=0.18 ; \mathrm{F}=11.0 ; \mathrm{p}<0.001$.

Table 3. Binary logistic regression analysis model

\begin{tabular}{|c|c|c|}
\hline & Odds Ratio and $95 \% \mathrm{CI}$ & $\mathbf{P}$ \\
\hline PTH status (either secondary hyperparathyroidism or functional hypoparathyroidism) & $1.81(1.11-2.95)$ & 0.017 \\
\hline Age & $1.02(0.99-1.05)$ & 0.143 \\
\hline Body Mass Index & $0.86(0.81-0.91)$ & $<0.001$ \\
\hline Phosphate (mg/dl) & $1.12(0.77-1.64)$ & 0.547 \\
\hline Albumin-adjusted calcium (mg/dl) & $1.15(0.92-1.44)$ & 0.212 \\
\hline 25-hydroxyvitamin D & $0.98(0.91-1.05)$ & 0.583 \\
\hline Estimated GFR & $1.01(0.99-1.02)$ & 0.093 \\
\hline Magnesium & $0.99(0.97-1.02)$ & 0.595 \\
\hline
\end{tabular}

The dependent variable was the presence of a low T-score (T-score $<-2.5$ at femoral neck assessment of bone mineral density) that was conventionally attributed a value of 1 (a T-score higher than -2.5 was conventionally attributed a value of 0 ). The independent variables included in the regression model are listed in the Table. Secondary hyperparathyroidism was conventionally attributed a value of 1 (functional hypoparathyroidism was conventionally attributed a value of 0 ). The full model was statistically significant $\left(\chi^{2}\right.$ $=45.7$;f $=8 ; \mathrm{P}<0.001)$. 
the women with secondary hyperparathyroidism had lower levels of BMD than those with normal PTH levels. Two previous reports have addressed the same issue, with conflicting results. ${ }^{16,18}$ Both the above studies investigated hip-fracture women with vitamin D depletion. Sahota et al. showed that hip BMD was lower in the women with PTH excess than in those with normal PTH levels, in agreement with our report. ${ }^{16}$ Conversely, Amaozugan et al. did not find any significant associations between PTH status and hip BMD.$^{18}$ The reasons for this discrepancy are not obvious. One possible explanation may be the presence of confounding factors. Indeed, several variables may play a confounding role by affecting PTH levels, BMD, or both. ${ }^{12,14,17,21}$ One strength of our study consists in the multiple adjustments we undertook: we took into account the role of age, BMI, phosphate, calcium, 25-hydroxyvitamin $\mathrm{D}$, estimated GFR, and magnesium, whereas in the two previous studies no adjustments were performed. ${ }^{16,18}$ One more strength of our study is sample size: we investigated 405 women, whereas Sahota et al. and Amazougan et al. studied 122 and 133 women, respectively. ${ }^{16,18}$

The link we show between secondary hyperparathyroidsm and reduced BMD is not surprising since a sustained elevation of PTH levels is known to exert catabolic effects on bone resulting in bone fragility. ${ }^{12,14}$ The clinical significance of our results rests on the excess of bone loss found in hip-fracture women with vitamin $\mathrm{D}$ depletion who developed secondary hyperaparathyroidism versus those who did not. Besides bone loss, other adverse consequences have been associated with PTH excess in hip-fracture patients, who are frail subjects ${ }^{7,8,22}$ with a high risk of recurrent falls ${ }^{23}$ and fractures, ${ }^{4,24}$ several comorbidities, ${ }^{22}$ and reduced life expectancy. ${ }^{25}$ The unfavorable outcomes associated with PTH excess include prolonged length of stay in hospital, ${ }^{26}$ reduced recovery in ability to function in activities of daily living, ${ }^{27}$ increased risk of being discharged to institutional care ${ }^{26}$ myocardial injury, ${ }^{26,28}$ and even all-cause mortality. ${ }^{26,28,29}$ The negative prognostic role of secondary hyperparathyroidism after hip-fracture is consistent with data in other groups of patients affected by various diseases, and even in the general aged population. ${ }^{30,31}$ Notably, PTH excess may worsen sarcopenia, ${ }^{32}$ which is highly prevalent in hip-fracture patients. ${ }^{33}$
Vitamin D deficiency was highly prevalent in our sample of hip-fracture inpatients, in agreement with the wider literature. ${ }^{7-11,16,18-21,26}$ We confirm that a substantial proportion of hip-fracture patients with severe vitamin D deficiency do not have secondary PTH elevation, ${ }^{16-20}$ though we did not elucidate the mechanisms underlying different PTH responses to vitamin D deficiency in different subjects. Magnesium depletion may play a pivotal role because it is associated with blunted PTH secretion, ${ }^{34,35}$ besides resistance to PTH action. ${ }^{36}$ We adjusted our data for serum magnesium levels, but they do not accurately reflect intracellular magnesium concentrations, which may be the actual determinants of PTH response..$^{35,37}$ Sahota et al, hypothesized other potential explanations to justify the blunted PTH response in individual subjects with vitamin D deficiency. ${ }^{38}$ Dysfunction of the parathyroid glands may be a potential cause and may include abnormalities of the parathyroid calcium sensing receptor, abnormalities of the serum 1,25-dihydroxyvitamin D receptor, or rarer causes such as abnormal expression of growth repressing genes within the gland, although these plausible hypotheses need to be supported by data. One more possible explanation for the inconsistent relationship between vitamin D deficiency and PTH excess could be the assessment of total and not free calcifediol concentration. In fact, similar concentrations of total 25-hydroxyvitamin D may result in different levels of free bioactive calcifediol, which in turn result in different PTH levels in the presence of different levels of binding proteins. ${ }^{39,40}$ Notably, following a hipfracture major changes in vitamin D-binding protein are expected because of both protein-depletion due to malnutrition ${ }^{41}$ and selective consumption due to the involvement of vitamin D-binding protein in the repair of injuried tissues. ${ }^{42}$

Our study has limitations. We evaluated one sample of Caucasian women who were surgically operated on and who were referred for inpatient rehabilitation. As a consequence, our results are not generalizable to the overall population of hip-fracture patients. In particular, the PTH response to vitamin D deficiency is different between men and women. ${ }^{20}$ Further studies should clarify the relationship between PTH levels and BMD in vitamin D depleted men. We did not collect data on some factors that could alter PTH 
levels, including dietary calcium intake and use of diuretics or corticosteroids. Seasonal variations in 25-hydroxyvitamin D levels were not investigated. Blood samples for laboratory assessment were collected around three weeks after fracture occurrence, thus a role of hospitalization in worsening the deficiency of vitamin D and modulating PTH response cannot be excluded. We assessed vitamin D status by measuring 25-hydroxyvitamin D levels. Although this is universally considered the best approach to assess an individual's vitamin D status, caveats, confounders, and controversies exist regarding this measurement. ${ }^{43}$ Finally, the cross-sectional design does not prove causal inference.

In conclusion, our results show that $\mathrm{PTH}$ response to vitamin $\mathrm{D}$ deficiency was significantly associated with femoral BMD in hip-fracture women. Prevention and treatment of vitamin D deficiency may be particularly relevant in those women who develop secondary hyperparathyroidism. Data from intervention trials with vitamin $\mathrm{D}$ supplements should be analyzed taking into account basal PTH levels which may affect the clinical benefits due to vitamin D supplementation.

\section{CONFLICT OF INTEREST}

On behalf of all authors, the corresponding author states that there is no conflict of interest.

The study was not funded. The authors have no conflicts of interest.

\section{REFERENCES}

1. Lieben L, Carmeliet G, Masuyama R, 2011 Calcemic actions of vitamin D: effects on the intestine, kidney and bone. Best Pract Res Clin Endocrinol Metab 25: 561-572.

2. Marcelli C, Chavoix C, Dargent-Molina P, 2015 Beneficial effects of vitamin $D$ on falls and fractures: is cognition rather than bone or muscle behind these benefits? Osteoporosis Int 26: 1-10.

3. Iolascon G, de Sire A, Calafiore D, Moretti A, Gimigliano R, Gimigliano F, 2015 Hypovitaminosis D is associated with a reduction in upper and lower limb muscle strength and physical performance in post-menopausal women: a retrospective study. Aging Clin Exp Res 27: Suppl 1: 23-30.

4. Kanis JA, McCloskey EV, Johansson H, Cooper C, Riz- zoli R, Reginster JY; Scientific Advisory Board of the European Society for Clinical and Economic Aspects of Osteoporosis and Osteoarthritis (ESCEO) and the Committee of Scientific Advisors of the International Osteoporosis Foundation (IOF), 2013 European guidance for the diagnosis and management of osteoporosis in postmenopausal women. Osteoporos Int 24: 23-57.

5. Van Schoor NM, Lips P, 2011 Worldwide vitamin D status. Best Pract Res Clin Endocrinol Metab 25: 671-680.

6. Katrinaki M, Kampa M, Margioris A, Castanas E, Malliaraki N, 2016 Vitamin D levels in a large Mediterranean cohort: reconsidering normal cut-off values. Hormones 15: 205-223.

7. Di Monaco M, Castiglioni C, Vallero F, Di Monaco R, Tappero R, 2011 Appendicular lean mass does not mediate the significant association between vitamin $\mathrm{D}$ status and functional outcome in hip fracture women. Arch Phys Med Rehabil 92: 271-276.

8. LeBoff MS, Hawkes WG, Glowacki J, Yu-Yahiro J, Hurwitz S, Magaziner J, 2008 Vitamin D-deficiency and post-fracture changes in lower extremity function and falls in women with hip fractures. Osteoporos Int 19: 1283-1290.

9. Bischoff-Ferrari HA, Can U, Staehelin HB, et al, 2008 Severe vitamin D deficiency in Swiss hip fracture patients. Bone 42: 597-602.

10. Ramason R, Selvaganapathi N, Ismail NH, Wong WC, Rajamoney GN, Chong MS, 2014 Prevalence of vitamin d deficiency in patients with hip fracture seen in an orthogeriatric service in sunny singapore. Geriatr Orthop Surg Rehabil 5: 82-86.

11. Di Monaco M, Castiglioni C, Di Monaco R, Tappero R, 2016 Time trend 2000-2013 of vitamin D status in older people who sustain hip fractures: steps forward or steps back? A retrospective study of 1599 inpatients. Eur J Phys Rehabil Med 52: 502-507.

12. Von Mühlen DG, Greendale GA, Garland CF, Wan L, Barrett-Connor E, 2005 Vitamin D, parathyroid hormone levels and bone mineral density in community-dwelling older women: the Rancho Bernardo Study. Osteoporos Int 16: 1721-1726.

13. Stein MS, Wark JD, Scherer SC, et al, 1999 Falls relate to vitamin D and parathyroid hormone in an Australian nursing home and hostel. J Am Geriatr Soc 47: 11951201.

14. Singhellakis PN, Malandrinou FC, Psarrou CJ, Danelli AM, Tsalavoutas SD, Constandellou ES, 2011 Vitamin $\mathrm{D}$ deficiency in white, apparently healthy, free-living adults in a temperate region. Hormones (Athens) 10: 131-143.

15. Lips P, 2001 Vitamin D deficiency and secondary hyperparathyroidism in the elderly: consequences for bone loss and fractures and therapeutic implications. Endocrine Rev 22: 477-501.

16. Sahota O, Gaynor K, Harwood RH, Hosking DJ, 2001 Hypovitaminosis D and 'functional hypoparathyroidism'- 
the NoNoF (Nottingham Neck of Femur) study. Age Ageing 30: 467-472.

17. Bjorkman MP, Sorva AJ, Risteli J, Tilvis RS, 2009 Low parathyroid hormone levels in bedridden geriatric patients with vitamin D deficiency. J Am Geriatr Soc 57: 1045-1050.

18. Amouzougan A, Chopin F, Laporte S, Vico L, Thomas T, 2011 Functional hypoparathyroidism in postmenopausal women with fragility fracture. Joint Bone Spine 79: 170-175.

19. Fisher A, Srikusalanukul W, Davis M, Smith P, 2010 Hip fracture type: important role of parathyroid hormone (PTH) response to hypovitaminosis D. Bone 47: 400-407.

20. Di Monaco M, Castiglioni C, Vallero F, Di Monaco R, Tappero R, 2013 Parathyroid hormone response to severe vitamin D deficiency is sex associated: an observational study of 571 hip fracture inpatients. J Nutr Health Aging 17: 180-184.

21. Di Monaco M, Castiglioni C, Vallero F, Di Monaco R, Tappero R, 2014 Parathyroid hormone variance is marginally explained by a panel of determinants: a cross sectional study of 909 hip-fracture patients. J Bone Miner Metab 32: 573-579.

22. Pioli G, Barone A, Mussi C et al; GIOG, 2014 The management of hip fracture in the older population. Joint position statement by Gruppo Italiano Ortogeriatria (GIOG). Aging Clin Exp Res 26: 547-553.

23. Di Monaco M, Vallero F, De Toma E et al, 2012 Adherence to recommendations for fall prevention significantly affects the risk of falling after hip fracture: post-hoc analyses of a quasi-randomized controlled trial. Eur J Phys Rehabil Med 48: 9-15.

24. Sosa M, Saavedra P, Gomez-de-Tejada MJ, et al, 2015 High prevalence of undiagnosed vertebral fractures in patients suffering from hip fracture at their hospital admission: weak concordance among observers. Aging Clin Exp Res 27: 835-839.

25. Abrahamsen B, van Staa T, Ariely R, Olson M, Cooper C, 2009 Excess mortality following hip fracture: a systematic epidemiological review. Osteoporos Int 20: 1633-1650.

26. Fisher A, Goh S, Srikusalanukul W, Davis M, 2009 Elevated serum PTH is independently associated with poor outcomes in older patients with hip fracture and vitamin D inadequacy. Calcif Tissue Int 85: 301-309.

27. Di Monaco M, Vallero F, Di Monaco R, Tappero R, Cavanna A, 2006 25-hydroxyvitamin D, parathyroid hormone, and functional recovery after hip fracture in elderly patients. J Bone Miner Metab 24: 42-47.

28. Fisher AA, Southcott EK, Srikusalanukul W, et al, 2007 Relationships between myocardial injury, all-cause mortality, vitamin D, PTH, and biochemical bone turnover markers in older patients with hip fractures. Ann Clin Lab Sci 37: 222-232.

29. Madsen CM, Jorgensen HL, Lind B, et al, 2012 Secondary hyperparathyroidism and mortality in hip fracture patients compared to a control group from general practice. Injury 43: 1052-1057.

30. Bjorkman MP, Sorva AJ, Tilvis RS, 2008 Elevated serum parathyroid hormone predicts impaired survival prognosis in a general aged population. Eur J Endocrinol 158: 749-753.

31. Domiciano DS, Machado LG, Lopes JB, et al, 2016 Bone mineral density and parathyroid hormone as Independent risk factors for mortality in community-dwelling older adults: A population-based prospective cohort study in Brazil. The São Paulo Ageing \& Health (spah) study. J Bone Miner Res 31: 1146-1157.

32. de Souza Genaro P, de Medeiros Pinheiro M, Szejnfeld VL, Martini LA, 2015 Secondary hyperparathyroidism and its relationship with sarcopenia in elderly women. Arch Gerontol Geriatr 60: 349-353.

33. Di Monaco M, Castiglioni C, De Toma E, et al, 2015 Presarcopenia and sarcopenia in hip-fracture women: prevalence and association with ability to function in activities of daily living. Aging Clin Exp Res 27: 465-472.

34. Rude RK, Singer FR, Gruber HE, 2009 Skeletal and hormonal effects of magnesium deficiency. J Am Coll Nutr 28: 131-141.

35. Sahota O, Mundey MK, San P, Godber IM, Hosking DJ, 2006 Vitamin D insufficiency and the blunted PTH response in established osteoporosis: the role of magnesium deficiency. Osteoporos Int 17: 1013-1021.

36. Yamamoto $M$, Yamaguchi T, Yamauchi M, Yano S, Sugimoto T, 2011 Acute-onset hypomagnesemia-induced hypocalcemia caused by refractoriness of bones and renal tubules to parathyroid hormone. J Bone Miner Metab 29: 752-755.

37. Zaloga GP, 1989 Interpretation of the serum Mg level. Chest 95: 257-258.

38. Sahota O, Mundey MK, San P, Godber IM, Lawson N, Hosking DJ, 2004 The relationship between vitamin D and parathyroid hormone: calcium homeostasis, bone turnover, and bone mineral density in postmenopausal women with established osteoporosis. Bone 35: 312-319.

39. Chun RF, Peercy BE, Orwoll ES, Nielson CM, Adams JS, Hewison M, 2014 Vitamin D and DBP: the free hormone hypothesis revisited. J Steroid Biochem Mol Biol 144: 132-137.

40. Powe CE, Evans MK, Wenger J, et al, 2013 Vitamin Dbinding protein and vitamin D status of black Americans and white Americans. N Engl J Med 369: 1991-2000.

41. Koren-Hakim T, Weiss A, Hershkovitz A, et al, 2012 The relationship between nutritional status of hip fracture operated elderly patients and their functioning, comorbidity and outcome. Clin Nutr 31: 917-921.

42. Meier U, Gressner O, Lammert F, Gressner AM, 2006 Gc-globulin: roles in response to injury. Clin Chem 52:1247-1253.

43. Binkley N, Wiebe D, 2013 Clinical controversies in vitamin D: $25(\mathrm{OH}) \mathrm{D}$ measurement, target concentration, and supplementation. J Clin Densitom 16: 402-408. 